Supplementary Information for

\title{
Pressure-Induced Optical Transitions in Metal Nanoclusters
}

Qi Li', Martín A. Mosquera², Leighton O. Jones², Abhinav Parakh³, Jinsong Chai, Rongchao Jin ${ }^{4}$, George C. Schatz ${ }^{2}, X$. Wendy $G u^{1 *}$

${ }^{1}$ Department of Mechanical Engineering, Stanford University, Stanford, CA 94305, United States

${ }^{2}$ Department of Chemistry, Northwestern University, Evanston, IL 60208, United States

${ }^{3}$ Department of Materials Science \& Engineering, Stanford University, Stanford, CA 94305, United States

${ }^{4}$ Department of Chemistry, Carnegie Mellon University, Pittsburgh, PA 15213, United States 


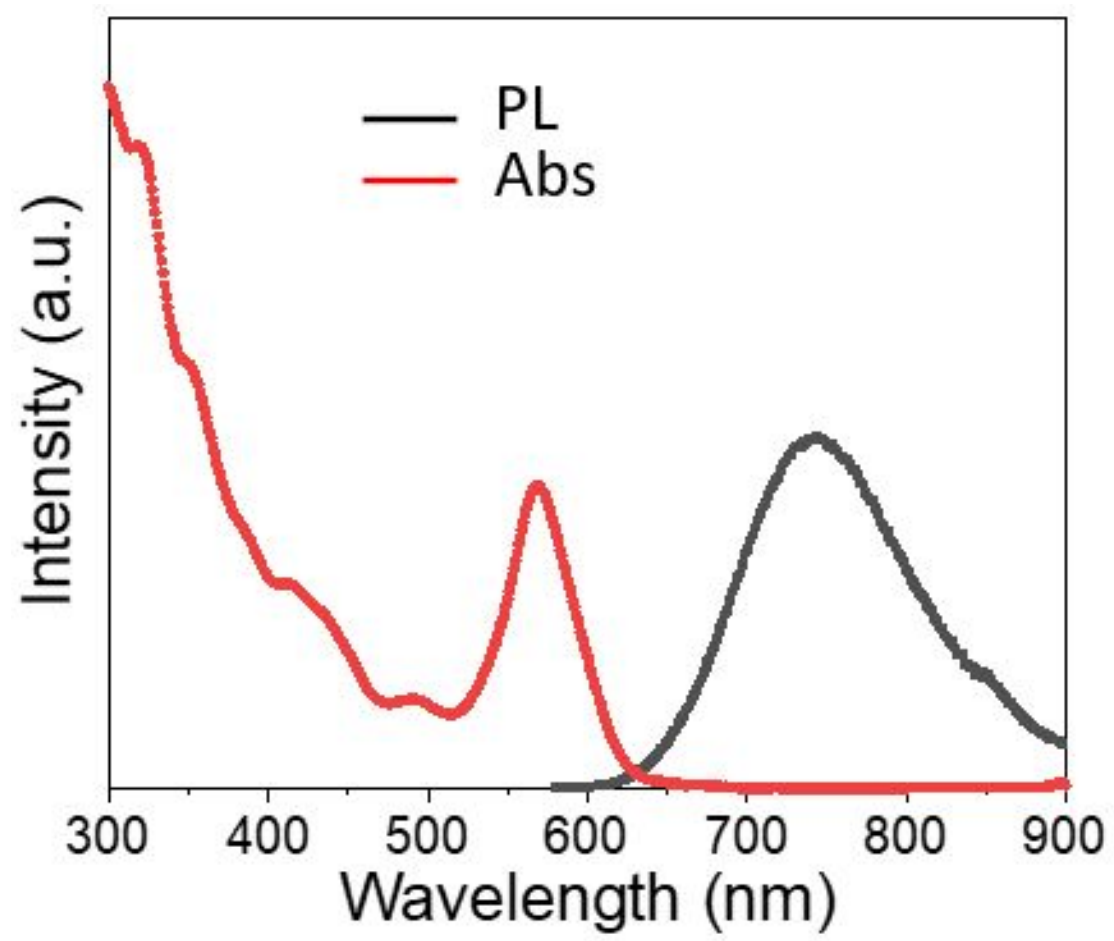

Figure S1. Absorption and PL of $\mathrm{Au}_{21}$ under ambient environment.
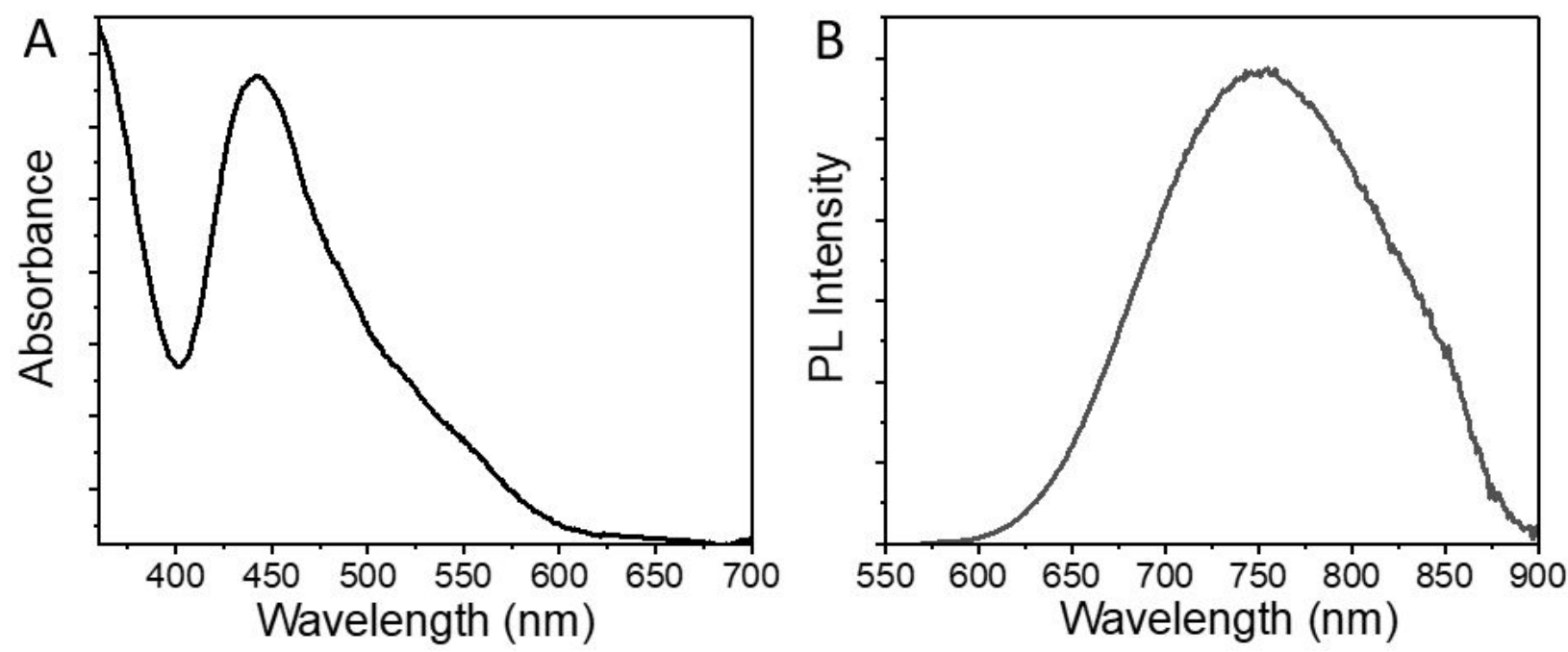

Figure S2. (A) Absorption and (B) PL of $\mathrm{Ag}_{28} \mathrm{Pt}_{1}$ under ambient environment. 


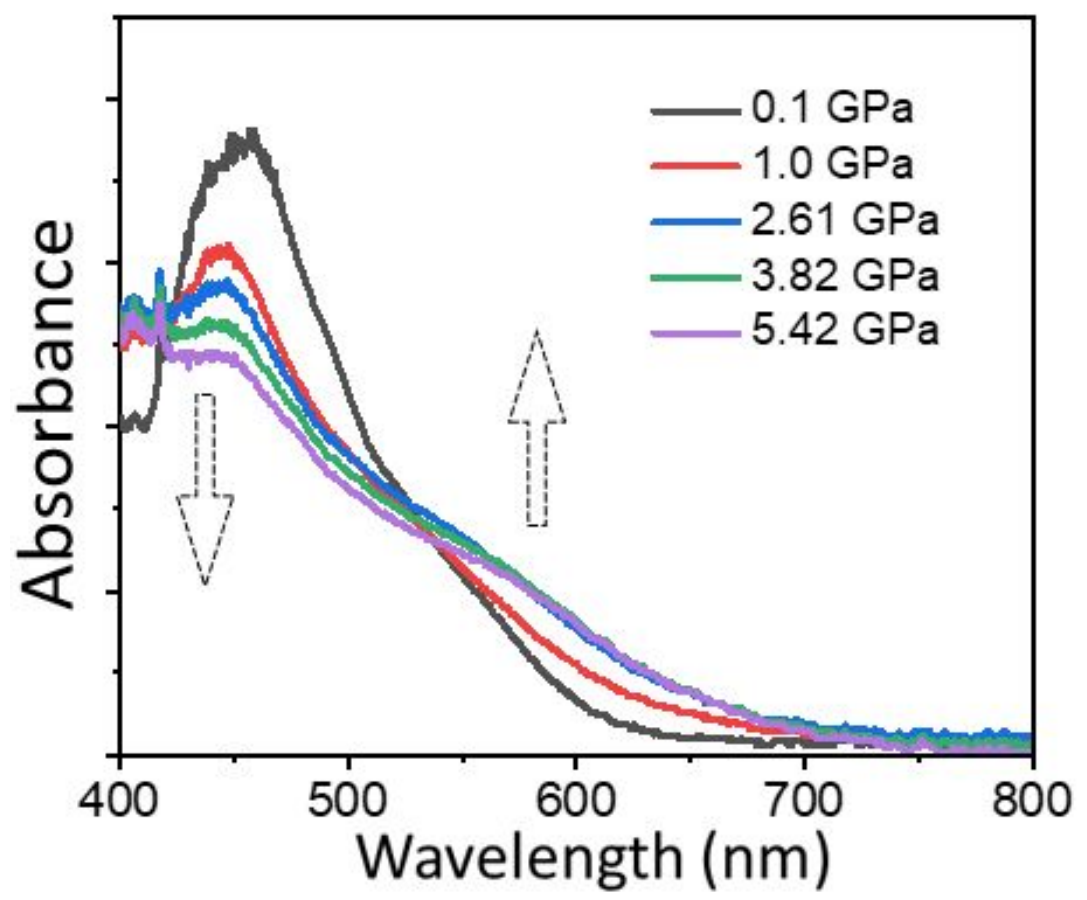

Figure S3. Pressure-dependent "ratiometric" response in the absorption of $\mathrm{Ag}_{28} \mathrm{Pt}_{1}$.

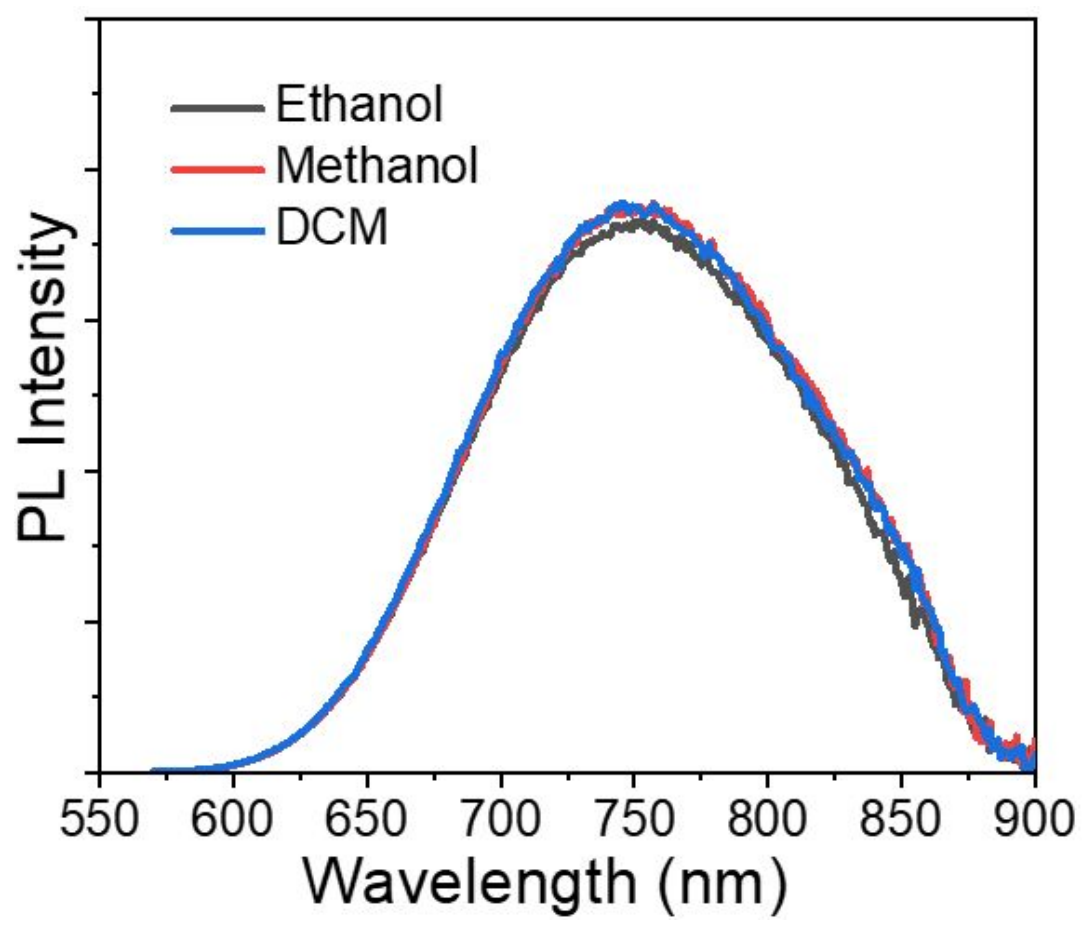

Figure S4. The solvent-dependent PL of $\mathrm{Ag}_{28} \mathrm{Pt}_{1}$ under ambient environment. 

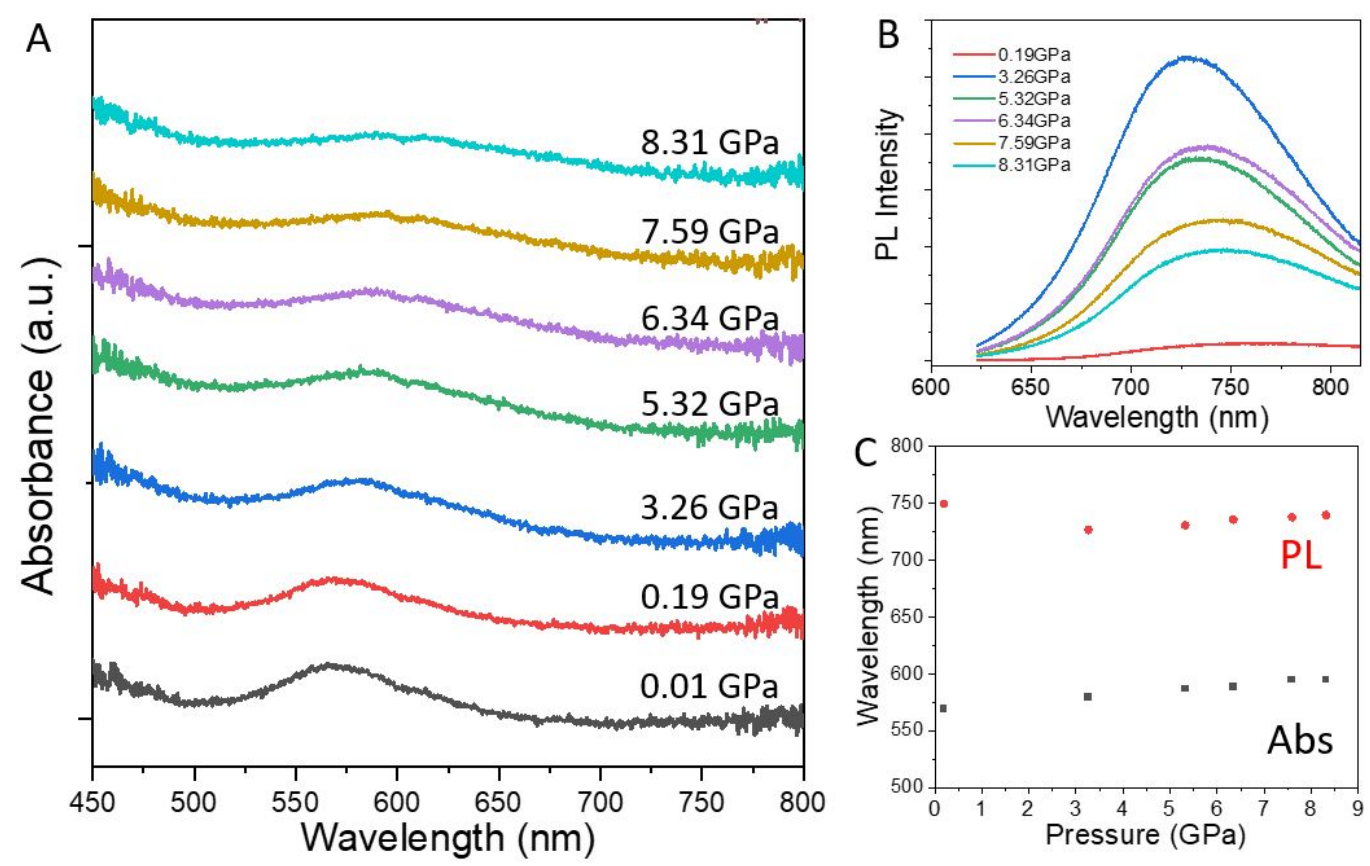

Figure S5. Pressure-dependent (A) absorption and (B) PL of $\mathrm{Au}_{21}$ in chloroform (non-hydrostatic). (C) Pressure-dependent evolutions of the absorption (black square) and PL (red dot) peak wavelengths.

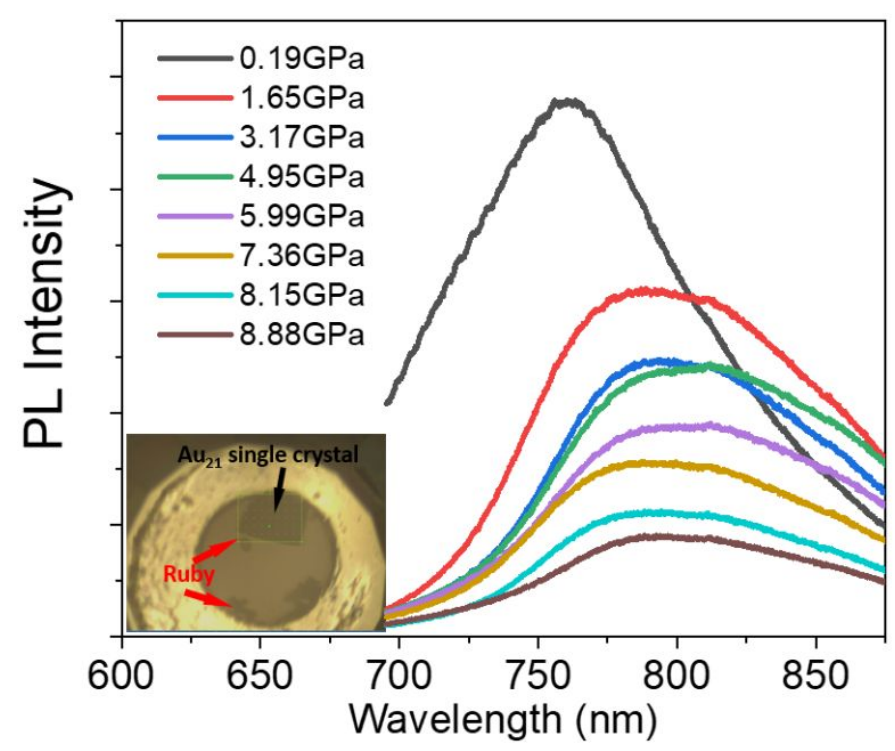

Figure S6. Pressure-dependent PL of $\mathrm{Au}_{21}$ crystal in 4:1 methanol/ethanol (quasi-hydrostatic medium). Inset is the photo of a $300 \mathrm{um}$ high pressure chamber which is filled with methanol/ethanol. A piece of $\mathrm{Au}_{21}$ single crystal and several pieces of ruby are loaded. The decrease of the PL intensity of $\mathrm{Au}_{21}$ nanocluster crystal under increasing pressures is probably due to the reduced inter-particle distances of nanoclusters in the close-packed crystals which could trigger non-radiative interparticle processes. 


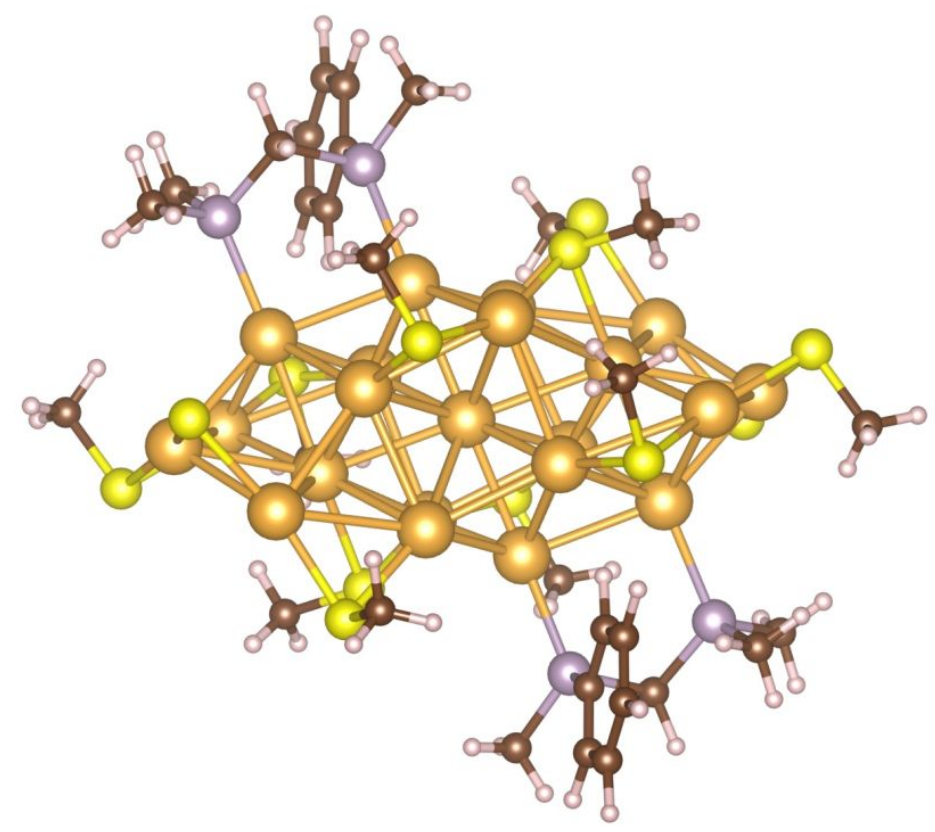

Figure S7. Isolated model of the $\mathrm{Au}_{21}$ by applying the common replacement of ligands by methyl substituents for simulation.

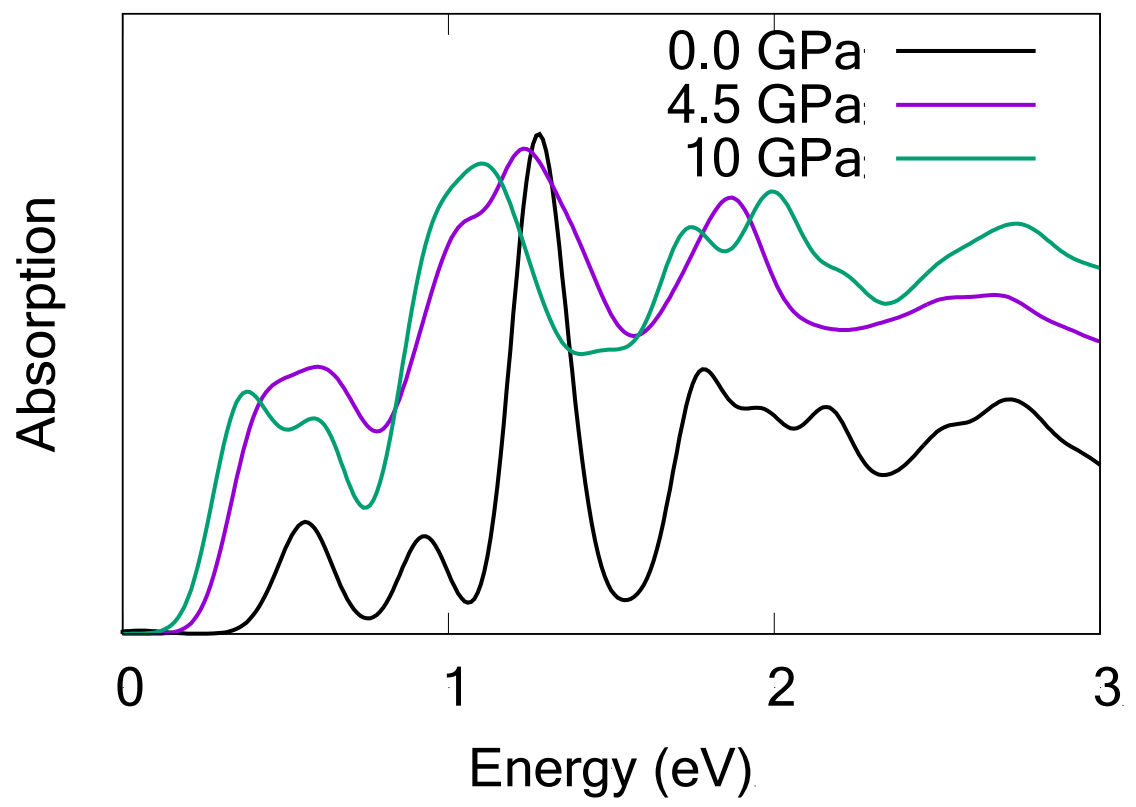

Figure S8. Computed absorption spectra of $\mathrm{Ag}_{28} \mathrm{Pt}_{1}$ nanocluster under different pressures. 

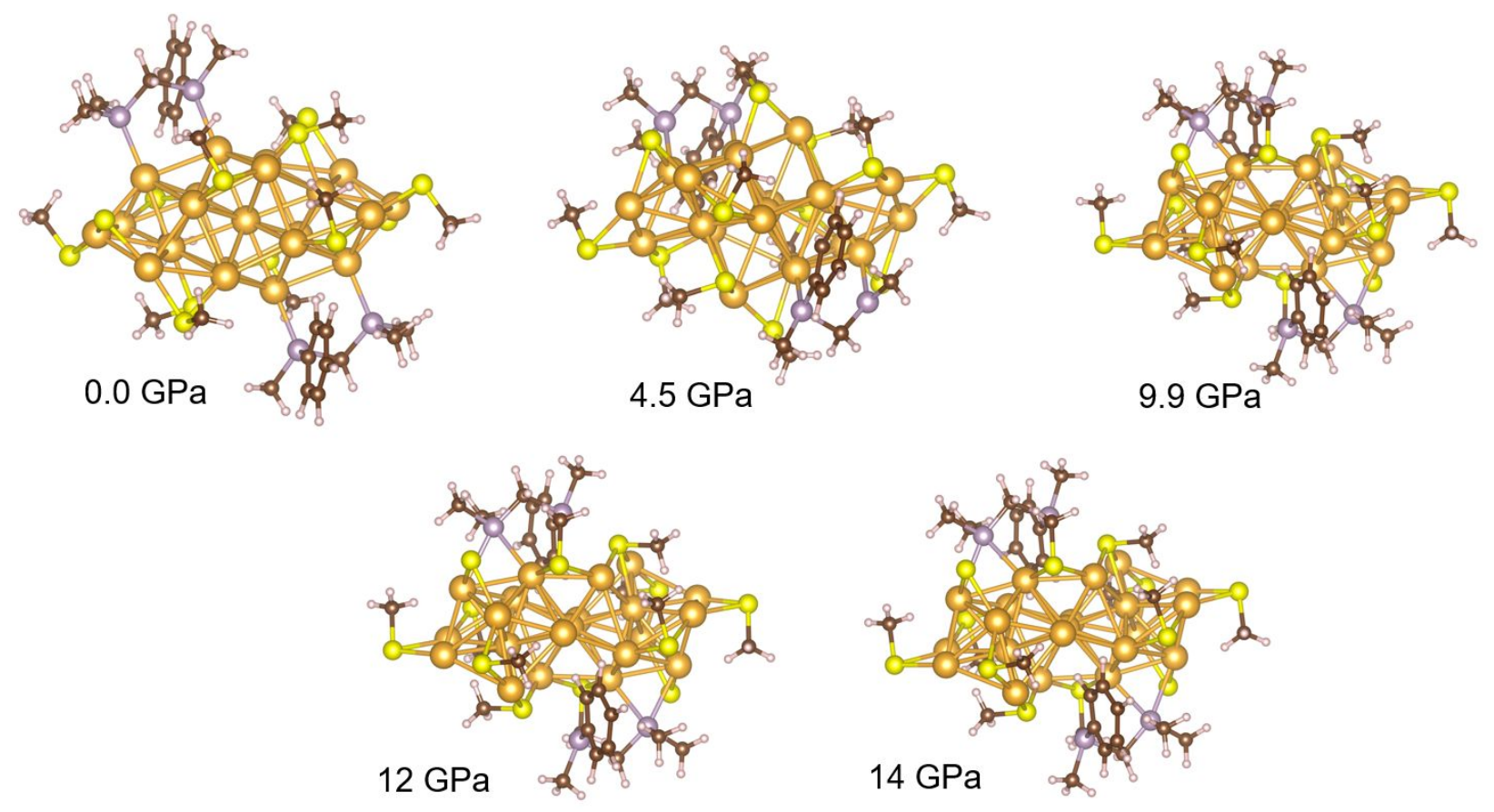

Figure S9. Simulated structures of the $\mathrm{Au}_{21}$ nanocluster under different pressures.

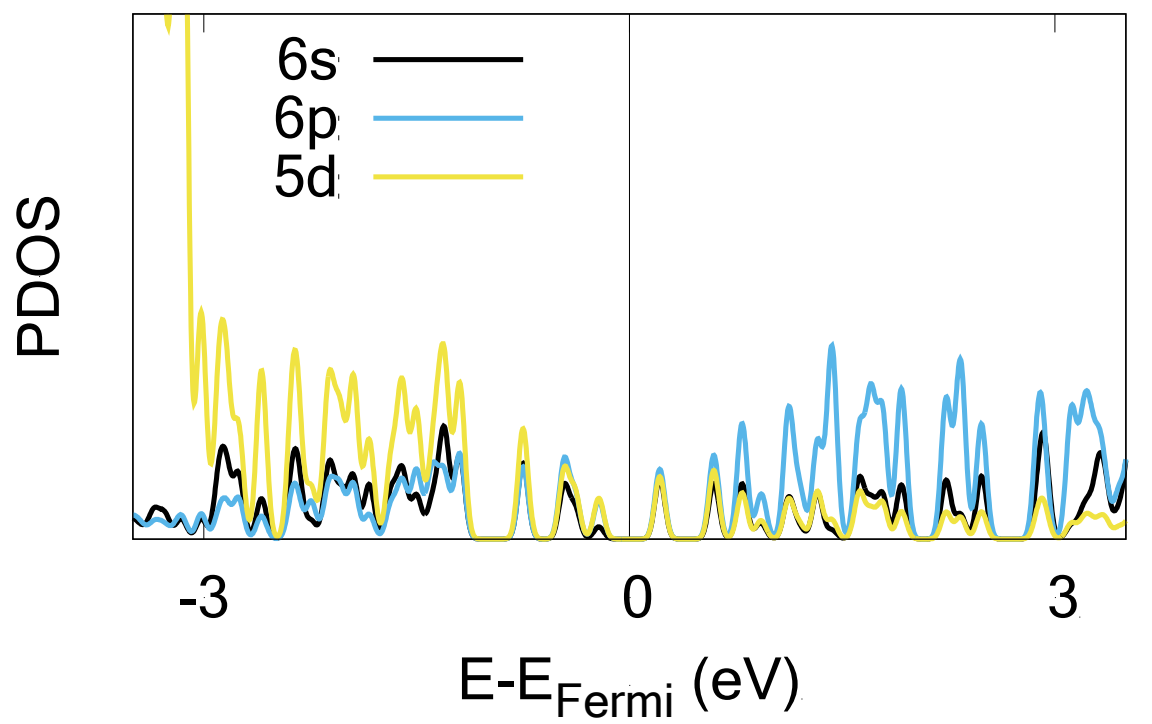

Figure S10. Projected density-of-states of the $\mathrm{Au}_{21}$ nanocluster under ambient environment. 

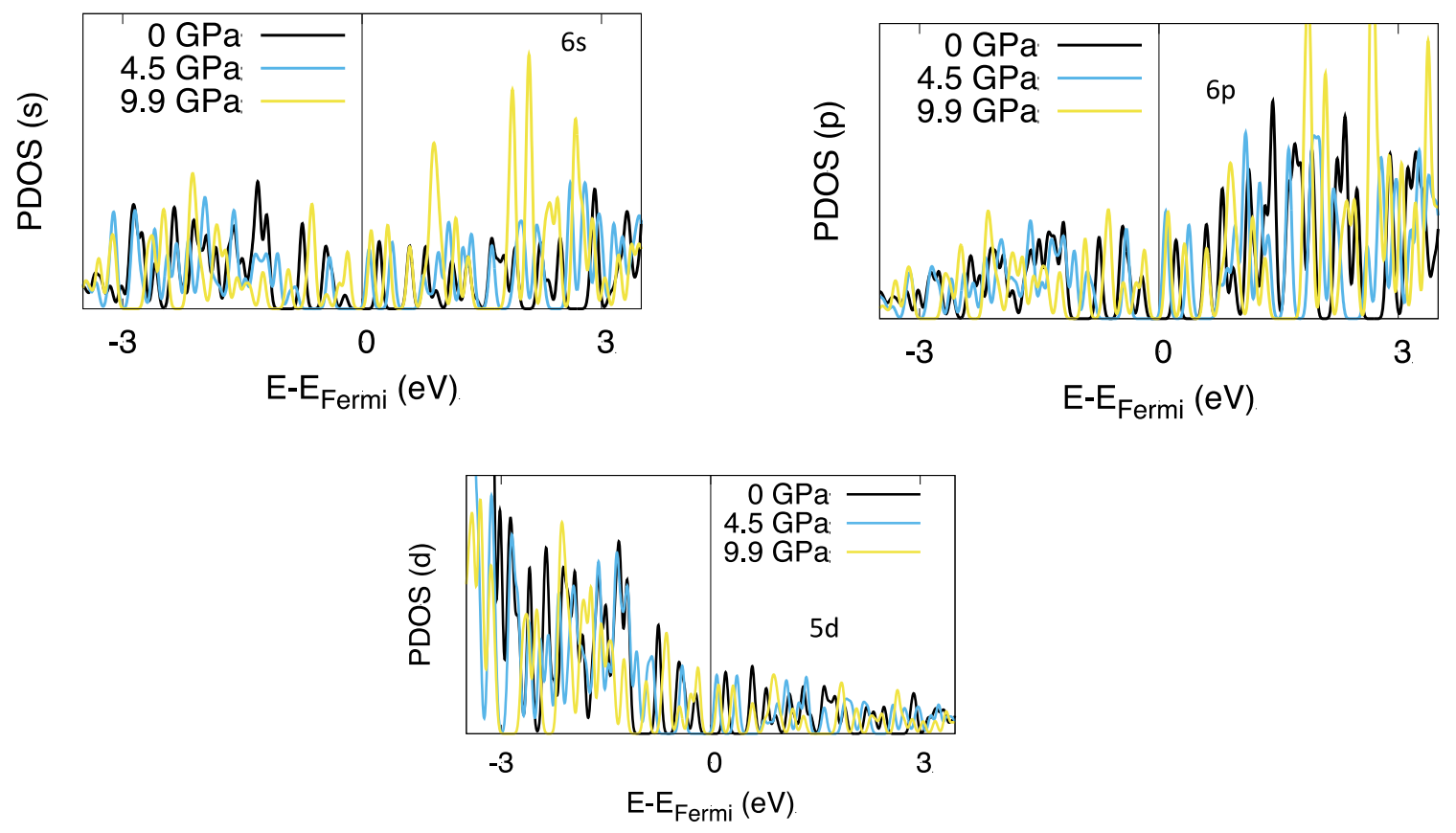

Figure S11. Projected density-of-states of the $\mathrm{Au}_{21}$ nanocluster under different pressures: $6 \mathrm{~s}$, $6 \mathrm{p}$, and $5 \mathrm{~d}$ PDOS shown.
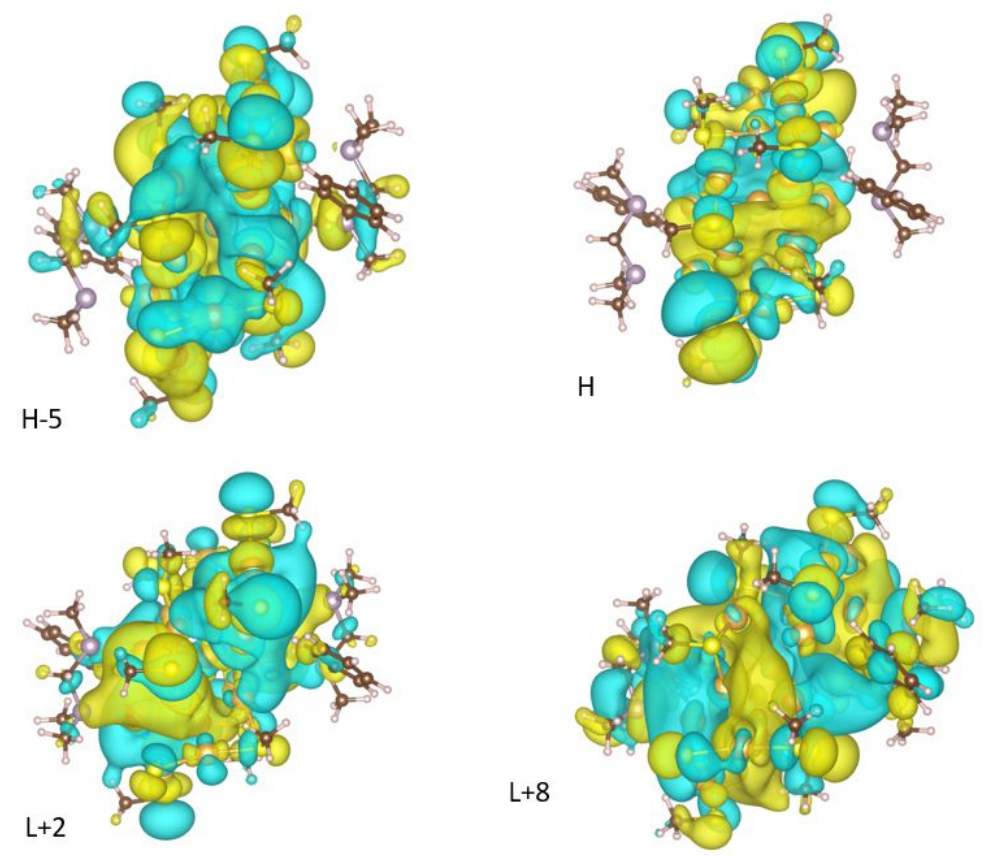

Figure S12. Frontier orbitals of the $\mathrm{Au}_{21}$ nanocluster under ambient environment. The $\mathrm{H}$ and $\mathrm{L}$ letters stand for highest occupied and lowest unoccupied molecular orbitals, respectively. 


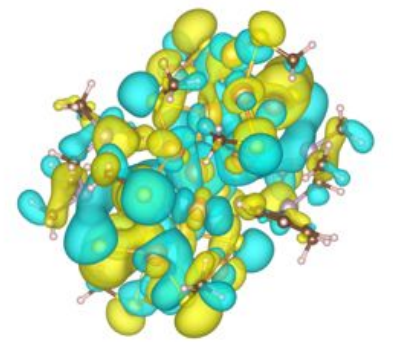

H-6

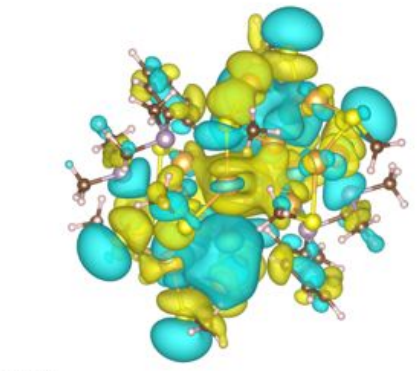

$L+2$

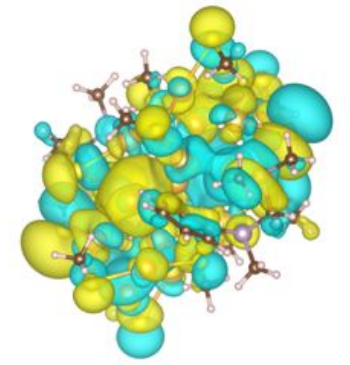

$\mathrm{H}$

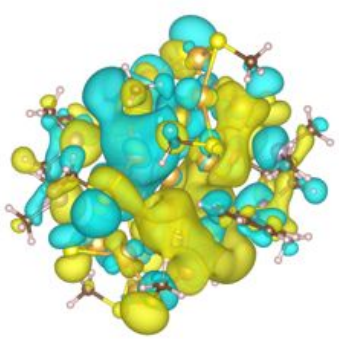

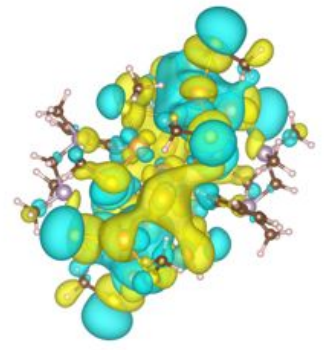

$\mathrm{L}$

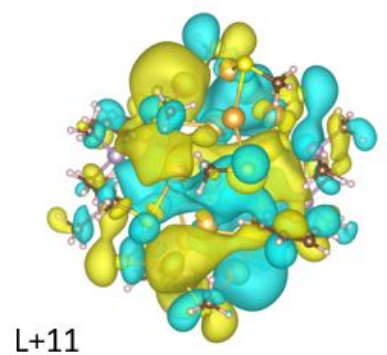

Figure S13. Frontier orbitals of the $\mathrm{Au}_{21}$ nanocluster under $9.9 \mathrm{GPa}$. The $\mathrm{H}$ and $\mathrm{L}$ letters stand for highest occupied and lowest unoccupied molecular orbitals, respectively.

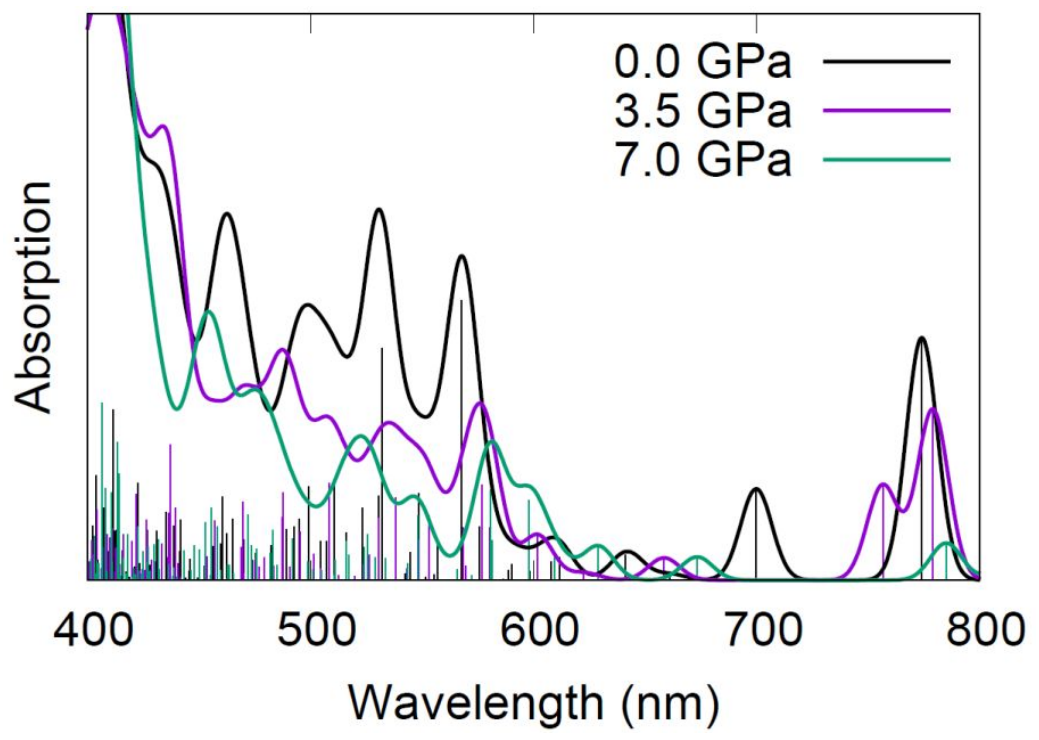

Figure S14. Computed absorption spectra of the $\mathrm{Au}_{14} \mathrm{Cd}$ nanocluster at different pressures. 
Tables S1. Calculated orbital contribution to the underlying transitions in the observed absorption peaks. Wavelength of the selected peak is listed below the pressure value.

\begin{tabular}{|l|c|c|c|c|c|c|c|c|c|c|}
\hline & \multicolumn{2}{|c|}{$\begin{array}{c}0 \mathrm{GPa} \\
652 \mathrm{~nm}\end{array}$} & \multicolumn{4}{c|}{$\begin{array}{c}4.5 \mathrm{GPa} \\
709 \mathrm{~nm}\end{array}$} & \multicolumn{2}{c|}{$\begin{array}{c}9.9 \mathrm{GPa} \\
796 \mathrm{~nm}\end{array}$} & \multicolumn{2}{c|}{$\begin{array}{c}12 \mathrm{GPa} \\
795 \mathrm{~nm}\end{array}$} \\
\hline & $\mathrm{H}-5---\mathrm{L}+2$ & $\mathrm{H}-1---$ & $\mathrm{L}+2$ & $\mathrm{H}-11$ & $\mathrm{~L}+1$ & \multicolumn{2}{c|}{$\mathrm{H}-6$--- $\mathrm{L}+3$} & $\mathrm{H}-6$--- L+3 \\
\hline $\mathrm{Au}_{6 \mathrm{~s}} \%$ & 16.2 & 39.5 & 17.1 & 54.1 & 14.8 & 35.3 & 18.3 & 60.1 & 21.0 & 61.2 \\
\hline $\mathrm{Au}_{6 \mathrm{p}} \%$ & 8.8 & 14.3 & 9.7 & 13.3 & 4.9 & 27.1 & 9.4 & 16.2 & 10.4 & 16.3 \\
\hline $\mathrm{Au}_{5 \mathrm{~d}} \%$ & 50.3 & 18.8 & 42.4 & 5.3 & 57.6 & 13.2 & 45.6 & 5.6 & 42.8 & 5.1 \\
\hline $\mathrm{S} \%$ & 20.8 & 23.0 & 22.6 & 7.4 & 14.2 & 16.7 & 19.5 & 8.9 & 18.8 & 7.8 \\
\hline $\mathrm{P} \%$ & 1.9 & 1.8 & 5.3 & 3.4 & 3.4 & 1.8 & 3.2 & 2.7 & 2.9 & 2.7 \\
\hline $\mathrm{C} \%$ & 2.0 & 2.6 & 2.9 & 16.5 & 5.1 & 5.9 & 4.0 & 6.5 & 4.1 & 6.9 \\
\hline
\end{tabular}

We selected a prominent absorption peak for each pressure and examine its dominant orbital transitions (based on the TDDFT calculations). To decompose these transitions into orbital contributions, for each molecular orbital, we squared the amplitude of each $6 \mathrm{~s}, 6 \mathrm{p}$, and $5 \mathrm{~d}$ orbital, and the valence $\mathrm{s}$ and $\mathrm{p}$ orbitals of the S, P, and C atoms. These contributions are normalized so they add up to $100 \%$. 